\title{
An intervention to improve discharge summary completion rates within an Australian teaching hospital
}

\author{
Fergus William Gardiner* \\ Calvary Health Care, Australia
}

Received: November 22, 2015

Accepted: December 9, 2015

Online Published: December 11, 2015

DOI: $10.5430 /$ jha.v5n2p1

URL: http://dx.doi.org/10.5430/jha.v5n2p1

\begin{abstract}
Objective: This study was designed to improve patient discharge summary completion rates directly following patient hospital discharge. The primary reason for this was to improve continuity of patient care and reduce hospital readmissions within 28 days. Methods: The researcher benchmarked the discharge summary completion rate before conducting individual feedback directly to clinicians. Content was deemed complete if the information was present and appropriate. Partially completed, unclear, or absent information was deemed outstanding. This information was gained by looking at the hospital's patient records. The researcher benchmarked the readmission data. This data included establishing monthly patient discharges (excluding deaths) and the number of unplanned and unexpected readmissions within 28 days related to the primary admission. This information was used to compare pre-intervention to invention readmission rates.

Results: The hospital's total discharge completion rate statistically changed from $91.92 \%$ pre-intervention to $99.18 \%$ postintervention, with the biggest change occurring in Obstetrics and Gynaecology $(\mathrm{O} \& \mathrm{G})$. O\&G discharge completion rate improved from $46.94 \%$ pre-intervention to $98.84 \%$ post-intervention. A two sample $t$-test indicated that this difference was significant, $t(2.0905)=0.0458, p=.05$. The readmission rates statistical changed from $0.49 \%$ pre-intervention to $0.26 \%$ during the intervention period. A two sample $t$-test indicated that this difference was significant, $t(2.3679)=0.04205, p=.05$.

Conclusions: This study provided evidence of the effectiveness of conducting audit and feedback sessions as it relates to patient discharge summaries and readmissions.
\end{abstract}

Key Words: Discharge summaries, Discharge planning, Audit and feedback, Medical education, Medical stewardship, Continuality of patient care, Patient readmission

\section{INTRODUCTION}

It has been well established that the transition from hospital care can present potential patient risks. ${ }^{[1-3]}$ Discharge summaries are important in limiting risk, and are used to communicate important information related to patient care that occurred within the hospitalization. ${ }^{[4]}$ The Australian Council on Healthcare Standards requires that discharge sum- maries are to be completed upon patient discharge. ${ }^{[5]}$ Many discharge summaries are ether incomplete or lacking important information. This can effect continuity of care and contribute to adverse events. ${ }^{[4]}$

Discharge summaries are especially relevant to general practitioners who do not have access to hospital patient records. A discharge summary normally includes information con-

*Correspondence: Fergus William Gardiner; Email: gus_gardiner@hotmail.com; Address: Calvary Health Care, Australia. 
cerning medications prescribed and any changes to current medications, as well as a list of current and inactive diagnoses, and any required follow-up. ${ }^{[6]}$ The Calvary Hospital Canberra requires that all discharge summaries include: patient identification, alerts, allergies and adverse reactions (if applicable), presenting problem, principal diagnosis, additional diagnosis (if applicable), procedures and investigations completed, significant incidents (if applicable), medications on admission and discharge (with reasoning associated with any changes), continued care recommendations, name, designation, and signature of the author, and the date finalised. It is recommended that only relevant information should be included.

There have been numerous attempts to improve the quality of discharge summaries. ${ }^{[7-9]}$ These attempts range from using structured paper formats to computer generated discharge systems. Calvary Hospital Canberra has a computer generated system which has demonstrated improvements in comprehensiveness and clarity, although even with this strategy, errors and delays in completion still occur. It has been determined that if discharge summaries are incomplete, delayed, or never written, then continuity of care can be negatively affected. It has been found that increased rates of re-admission to the hospital are associated with delays in discharge summary finalization, and that $11 \%$ of patient discharges have a preventable adverse event occurring post-hospital discharge, with many of these events related to inadequate communication between the hospital and external medical care. The absence of a discharge summary has been associated with a $79 \%$ increase in hospital readmission within 28 days. ${ }^{[6]}$

It has been suggested that direct individualised feedback can improve the quality and completion rates of discharge summaries. ${ }^{[10]}$ Furthermore, to test whether adequately completed discharge summaries are associated with decreased hospital readmission rates, ${ }^{[6]}$ the researcher tested the following research questions:

Hypothesis 1: Individualized feedback improves the rates of discharge summary completion in targeted hospital areas.

Hypothesis 2: Individualized feedback targeting the rates of discharge summary completion correlates to a decreased patient readmission rate within 28 days.

\section{MethodS}

\subsection{Setting}

The researcher benchmarked the Calvary Hospital Canberra's discharge summary completion rates between 16/08/2015 and $16 / 09 / 2015$, before conducting an intervention between $17 / 09 / 2015$ and 16/11/2015.

\subsection{Participants}

Included were all clinicians who are required to complete discharge summaries within the hospital, excluding the Emergency Department. This included the admitting senior doctor and their delegated junior medical officers.

\subsection{Pre-intervention audit}

All discharge summaries were audited for completeness between 16/08/2015 and 16/09/2015 ( $\mathrm{N}=789)$, the day directly after the patient was discharged. Content was deemed complete if the information was present and appropriate to the clinical diagnosis $(\mathrm{N}=732)$. Partially completed, unclear, or absent information was deemed inappropriate and outstanding $(\mathrm{N}=57)$. This information was gained by looking at the patient record. This intervention highlighted Obstetrics and Gynaecology $(\mathrm{O} \& \mathrm{G})$ as the clinical area with the lowest completion rate at $46.94 \%(\mathrm{~N}=26)$.

Auditing of the patient records was completed ether in the applicable clinical area, or via accessing the medical records department. Patient discharge data was gained via the hospital data unit, and the patient flow unit.

\subsection{Intervention}

The researcher between 17/09/2015 and 16/11/2015 conducted daily audits of patient notes, the day directly after the patient was discharged, before conducting one-on-one formative feedback directly to clinicians with discharge summaries deemed outstanding during this period $(\mathrm{N}=114)$. As with the pre-intervention, audited discharge summary content was deemed complete if the information was present and appropriate. Partially completed, unclear, or absent information was deemed outstanding.

The feedback sessions lasted approximately 5 minutes. During these sessions each clinician received the results of their discharge summary audit. Each error was discussed and the importance of completed discharge summaries was highlighted. The individual "clinical outstanding discharge summary report" data has not been included in this article due to confidentiality. In the case where the clinician was not available, the researcher conducted the formative feedback to their direct senior. This included a combination of one-on-one discussions, emails, and phone messages.

\subsection{Hospital readmission rates}

To determine whether audit and feedback interventions had an effect on hospital readmission rates, the researcher benchmark the hospitals readmission data from January until August 2015 was collected and compared this to the intervention months of September, October, and November 2015. This data included establishing monthly patient discharges 
(excluding deaths) and the number of unplanned and unexpected readmissions within 28 days related to the primary admission.

\subsection{Statistical analysis}

A two sample $t$-test was used to compare the non-intervention period to the intervention period. A significance of .05 was used.

\section{RESUlTS}

\subsection{Post-intervention audit results}

All discharge summaries, during the intervention period, were audited for completeness at the conclusion of the inter- vention on $17 / 11 / 2015(\mathrm{~N}=1,471)$. The total discharge completion rate statistically changed from $91.92 \%$ preintervention to $99.18 \%$ post-intervention, with the biggest change occurring in $\mathrm{O} \& \mathrm{G}$. O\&G discharge completion rates improved from $46.94 \%$ pre-intervention to $98.84 \%$ postintervention. A two sample $t$-test indicated that this difference was significant, $t(2.0905)=0.0458, p=.05$ (see Tables 1 and 2).

Results indicate that individualised feedback improves the rates of discharge summary completion. These results could reflect that individualised feedback to speciality areas improves the rates of discharge summary completion.

Table 1. Pre-intervention hospital data (discharge summary completion rates 16/08/2015- 16/09/2015)

\begin{tabular}{|c|c|c|c|c|}
\hline Team & $\begin{array}{l}\text { Discharges requiring } \\
\text { discharge summary }\end{array}$ & Completed & Uncompleted & Completion rate \\
\hline Age Care and Rehabilitation & 16 & 15 & 1 & $93.75 \%$ \\
\hline Cardiology & 51 & 42 & 9 & $82.35 \%$ \\
\hline Floating Medical Team & 9 & 8 & 1 & $88.89 \%$ \\
\hline Medical Assessment and Planning Unit & 152 & 151 & 1 & $99.34 \%$ \\
\hline Medical team 1 & 40 & 40 & 0 & $100.00 \%$ \\
\hline Medical team 3 & 49 & 48 & 1 & $97.96 \%$ \\
\hline Medical team 4 & 56 & 54 & 2 & $96.43 \%$ \\
\hline Obstetrics and Gynaecology & 49 & 23 & 26 & $46.94 \%$ \\
\hline Operating room/theatres & 50 & 50 & 0 & $100.00 \%$ \\
\hline Stroke Service & 27 & 26 & 1 & $96.30 \%$ \\
\hline Surgical team 1 & 56 & 54 & 2 & $96.43 \%$ \\
\hline Surgical team 3 & 75 & 66 & 9 & $88.00 \%$ \\
\hline Surgical team 4 & 56 & 55 & 1 & $98.21 \%$ \\
\hline Grand Total & 789 & 732 & 57 & $91.92 \%$ \\
\hline
\end{tabular}

Table 2. Post-intervention hospital data (discharge summary completion rates 17/11/2015)

\begin{tabular}{|c|c|c|c|c|}
\hline Team & $\begin{array}{l}\text { Discharges requiring } \\
\text { discharge summary }\end{array}$ & Completed & Uncompleted & Completion rate \\
\hline Age Care and Rehabilitation & 38 & 38 & 0 & $100.00 \%$ \\
\hline Cardiology & 135 & 133 & 2 & $98.52 \%$ \\
\hline Floating Medical Team & 28 & 28 & 0 & $100.00 \%$ \\
\hline Medical Assessment and Planning Unit & 296 & 293 & 3 & $98.99 \%$ \\
\hline Medical team 1 & 79 & 77 & 2 & $97.47 \%$ \\
\hline Medical team 2 & 98 & 98 & 0 & $100.00 \%$ \\
\hline Medical team 3 & 92 & 91 & 1 & $98.91 \%$ \\
\hline Medical team 4 & 91 & 91 & 0 & $100.00 \%$ \\
\hline Obstetrics and Gynaecology & 86 & 85 & 1 & $98.84 \%$ \\
\hline Operating room/theatres & 74 & 74 & 0 & $100.00 \%$ \\
\hline Stroke Service & 33 & 33 & 0 & $100.00 \%$ \\
\hline Surgical team 1 & 109 & 108 & 1 & $99.08 \%$ \\
\hline Surgical team 3 & 111 & 110 & 1 & $99.10 \%$ \\
\hline Surgical team 4 & 85 & 83 & 2 & $97.65 \%$ \\
\hline Grand Total & 1471 & 1457 & 14 & $99.18 \%$ \\
\hline
\end{tabular}




\subsection{Hospital re-admission rates}

To test whether feedback and auditing had an effect on hospital readmission rates, the researcher compared the pre-intervention percentage of hospital readmissions to the intervention period $(0.49 \%$ vs. $0.26 \%)$. A two sample $t$-test indicated that this difference was significant, $t(2.3679)=0.04205, p=.05$.

\section{Discussion AND CONCLUSION}

This study demonstrates that audit and feedback interventions improve completion rates of discharge summaries. The pre-intervention audit demonstrated that completeness was satisfactory in most areas, although unacceptable in others critical for safe patient transition into community care. These findings were not surprising due to feedback received from external medical officers about delayed discharge summaries related to specific hospital areas. During feedback sessions, junior medical officers suggested low completion rates were due to a combination of staffing shortfalls, not prioritising discharge summaries, and a lack of understanding of the importance of completing the discharge summary promptly and in its entirety.

The feedback sessions had a dramatic effect on completion rates, with rates drastically improving directly after consultation. It is believed that by notifying the medical officer directly, and by providing detailed reporting, the medical officers' awareness of the problem was improved and motivated a change in practice. The audit and feedback process was not difficult, or time consuming, and directly improved continuality of care through improved discharge summary completion rates.

This study demonstrates that prompt and completed discharge summaries appear to be associated with a reduction in hospital readmissions rates. This is consistent with other Australian research, demonstrating an association between delayed transmission or absence of a discharge summary and readmission rates. ${ }^{[6]}$ It is unclear whether a causal relationship can be established, although results are promising.
The intervention had a debatable time commitment. The researcher argues the time commitment justified the outcomes. The researcher spent an estimated 3-4 (working) weeks conducting audit and feedback over a 3 month period. Specifically, the researcher estimated that between 15 and 17 patient notes (1.0-1.5 hours) were audited a day, before conducting between 1.5-2.0 hours of feedback. Due to the debateable time cost associated with these results, it is unknown whether this intervention will appeal to all hospitals, although it is argued that the time expense justified the outcomes via improved patient continuity of care. Furthermore, it is believed the reduced readmissions rates benefited the hospital financially through a reduction in patient bed days. Results could be reproduced by other hospital clinicians looking to improve discharge summary completion rates within their area. Specifically, feedback could be given to junior medical officers during handover, clinical rounding, or during formalised meetings.

This study had limitations, including the intervention was limited to one case hospital, and the rotating nature of the junior medical officers. This intervention will need to be reinforced to new medical rotations.

This study provided evidence of the effectiveness of conducting audit and feedback sessions as it relates to discharge summaries. This outcome provided improved continuity of patient care through reduced re-admission rates. Furthermore, as a teaching hospital it highlights the effectiveness of non-traditional educational modalities.

Future research topics could include determining the financial impact of reduced readmissions, which clinical outcomes most correlated with readmissions, and the potential impact of using electronic health records with associated alerts on completion of discharge summaries.

\section{CONFlicts of INTEREST Disclosure}

The author would like to acknowledge he is an employee of Calvary Health Care.

\section{REFERENCES}

[1] Forster AJ, Murff HJ, Peterson JF, et al. The incidence and severity of adverse events affecting patients after discharge from hospital. Annals of Internal Medicine. 2003; 138(3): 161-7. PMid: 12558354. http://dx.doi.org/10.7326/0003-4819-1 38-3-200302040-00007

[2] Easton K, Morgan T, Williamson M. Medication safety in the community: a review of the literature. Sydney: National Prescribing Service; 2009.
[3] Tam VC, Knowles SR, Cornish PL, et al. Requency, type and clnical importance of medication history errors at admission to hospital: a systematic review. CMAJ. 2005; 173(5): 510-5. PMid: 16129874. http://dx.doi.org/10.1503/cmaj.045311

[4] Lehnbom EC, Raban MZ, Walter SR, et al. Do electronic discharge summaries contain more complete medication information? A retrospective analysis of paper versus electronic discharge summaries. HIMJ. 2014; 43(3): 4-12.

[5] Publications and Resources: The Australian Council on Healthcare 
Standards; 2015. Available from: http://www .achs.org.au/pu blications-resources/equipnational/

[6] Li JY, Yong TY, Hakendorf P, et al. Timeliness in discharge summary dissemination is associated with patients' clinical outcomes. J Eval Clin Pract. 2013; 19(1): 76-9. PMid: 21999337. http: //dx.doi.org/10.1111/j.1365-2753.2011.01772.x

[7] Archbold RA, Laji K, Suliman A, et al. Evaluation of computergenerated discharge summary for patients with acute coronary syndromes. PubMed. 1998; 48(429): 1163-4.

[8] Castleden W, Stacey M, Norman P, et al. General practitioners' at- tidudes to computer-generated surgical discharge letters. Med J Aust. 1992; 157(6): 380-2. PMid: 1447986.

[9] O’Leary K, Liebovitz D, Feinglass J, et al. Creating a better discharge summary: improvement in quality and timeliness using an electronic discharge summary. J Hops Med. 2009; 4(4): 219-25. http://dx.doi.org/10.1002/jhm. 425

[10] Dinescu A, Fernandez H, Ross JS, et al. Audit and feedback: an intervention to improve discharge summary completion. J Hosp Med. 2011; 6(1): 28-32. PMid: 21241038. http://dx.doi.org/10.10 $02 / \mathrm{jhm} .831$ 